\title{
A Functional Comparison of the Archaeon Tricorn Protease and Selected Serine Proteases from Trypanosoma brucei brucei
}

\author{
Ng'ong'a, F.A ${ }^{1}$, Wamunyokoli, $F^{2}$, Nyanjom, S. R. G ${ }^{3}$ \\ ${ }^{1,2,3}$ Department of Biochemistry, Jomo Kenyatta University of Agriculture and Technology, P. O. Box 62000-00200, Nairobi, Kenya
}

\begin{abstract}
Trypanosomes are protozoan parasites causing African trypanosomiasis, a neglected tropical disease in Africa affecting humans and animals. Current control methods have focused on the use of drugs which have adverse effects and develop resistance and with no available conventional vaccine. Proteolysis is a key process in trypanosome survival in the mammalian host hence identification of other parasitic factors would lead to the development of new chemotherapeutic agents. This study investigated archaeon tricorn protease functional analogs in Trypanosoma brucei brucei through bioinformatics approaches. The protein sequences were retrieved from NCBI (http://www.ncbi.nlm.nih.gov/protein/) and position specific iterated basic local sequence alignment (PSI-BLAST) was performed using default parameters to determine patterns of conservation which aid in the recognition of distant similarities. The $3 D$ models of the putative proteins were constructed using T. acidophilum tricorn protease. The constructed models were analyzed based on percentage identity, e-value and bit-score. Structural alignment was done using MATRAS (http://strcomp.protein.osaka-u.ac.jp/matras/) and PyMOL Molecular Graphics System, Version 1.8 Schrödinger, LLC (https://www.pymol.org/) was used for viewing and structural analysis. The bioinformatics analysis revealed similarities in the catalytic core elements as well as the beta sheets serving as substrate entry and exit route to and from the catalytic chamber. Therefore, based on these similarities, this study reports the identification tricorn protease functional analogs in Trypanosoma brucei brucei.
\end{abstract}

Keywords: Tricorn protease, trypanosomiasis, protein homology, serine peptidases, proteolysis

\section{Overview}

African Trypanosomiasis is a vector-borne disease caused by protozoan parasites of the genus Trypanosoma (Franco et al. 2014). There are two forms of the disease; Human African Trypanosomiasis (HAT) caused by T.b gambiense and T.b rhodensiense and Animal African Trypanosomiasis (AAT) caused by $T$. b. brucei, T. congolense, T. vivax among others. Wild and domestic animals can host the parasite hence act as a reservoir of infection for tsetse flies (Franco et al. 2014). The disease control strategies have relied on the use of drugs which have adverse effects and vector control methods which have proved in efficient (Simo et al. 2014). The development of a convectional vaccine has been hampered by the ability of the parasite to express variable surface glycoproteins (Simo et al. 2014). $T$. $b$. brucei is unable to infect primates due to its susceptibility to lysis by the human Trypanosome Lysis Factor-1 and is genotypically similar to the human pathogenic forms to $T$. $b$. gambiense and T. $b$. rhodensiense hence making it a good experimental model for human and animal infections studies (Simarro et al. 2011).

Initial intracellular protein degradation in the archaeon Thermoplasma acidophilium, is carried out by proteasome employing sieving mechanisms for substrate selection (Bochtler et al. 1999). Products of proteasomes are peptides of about 6-12 amino acid residues which are further degraded by tricorn protease and its interacting factors thus completing the proteasomal degradation pathway (Tamura et al. 1996). The C-terminal of tricorn protease consists of $\mathrm{C} 1$, PDZ,C2 domains and harbors the active site residues (S745, H746, S965, E 1023) (Brandstetter et al. 2001). Unlike the archaeon proteasome, the molecular protein degradation machinery in trypanosomes is thought to have trypsin-like, chymotrypsin-like and caspase-like proteolytic activity (Cardoso et al. 2011). This enables the trypanosome to degrade an array of peptides hence a vast products for further processing.

In this study, a structural analysis of tricorn protease was done with a view of identifying similarities with some $T . b$. brucei cytosolic serine proteases. The structural prediction of the putative T. b. brucei sequences was done based on homology modeling. Domain organization and composition were subsequently analyzed with focus on the active site residues and thus the study reports the functional similarities of tricorn protease and T. b. brucei serine proteases.

\section{Methods}

Sequence retrieval and analysis

The T. acidophilum tricorn protease (ACC4462.1) and the putative $T . b$. brucei protein sequences (EAN78104.1, EAN80234.1 and EAN78208.1) were retrieved from NCBI database (https://www.ncbi.nlm.nih.gov/). Sequence similarity searches were conducted in NCBI at blast.ncbi.nlm.nih.gov/Blast.cgi (Altschul et al. 1990).The sequences were analyzed through MAFFT v7 (http://mafft.cbrc.jp/alignment/server/index.html) (Katoh \& Standley 2013) multiple sequence alignment tool to determine the conserved residues and viewed in Jalview (Waterhouse et al. 2009).

\section{Protein modeling and analysis}

The 3D models of the T. brucei brucei proteins were constructed using (PS)v2 (http://ps2.life.nctu.edu.tw/) (Huang et al. 2015). The constructed models were analyzed based on percentage identity, e-value and bit-score and 


\section{International Journal of Science and Research (IJSR) \\ ISSN (Online): 2319-7064 \\ Index Copernicus Value (2013): 6.14 | Impact Factor (2015): 6.391}

saved as pdb files. Structural alignment was done using MATRAS (http://strcomp.protein.osaka-u.ac.jp/matras/) (Kawabata 2003). PyMOL Molecular Graphics System, Version 1.8 Schrödinger, LLC (https://www.pymol.org/) (Delano, 2002) was used for viewing and structural analysis.

\section{Results and Discussion}

The PSI-BLAST results revealed a weak homology between tricorn protease and $T . \quad b$. brucei proteins including dipeptidyl peptidase IV (Tb927.10.6940), prolyl oligopeptidase (Tb927.10.8020) and oligopeptidase B (Tb927.11.12580. Structural analysis of the proteins revealed similarity with tricorn protease in that their Cterminal regions which harbour the active site residues (Figure 2a). These serine proteases also belong to the alphabeta hydrolases. In all the structures, the catalytic core element (serine) was strictly conserved and was shown to lie at the entrance of a conserved helix (Figure 2b). The orientation of tricorn's 6- and 7-bladed beta propeller domains along the polypeptide seem to be similar to the orientation of the beta propeller sheets of the trypanosome proteins and both have been shown to act as channels for substrate exit and entry to the active site (Figure 2b). The active site residues were also oriented in a similar fashion along the polypeptide chain (Figure 2c).
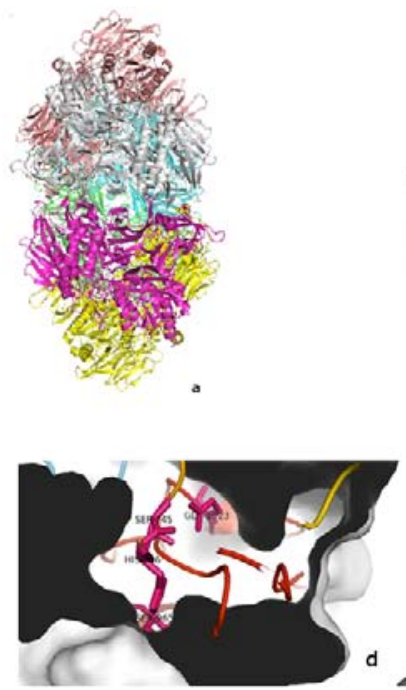
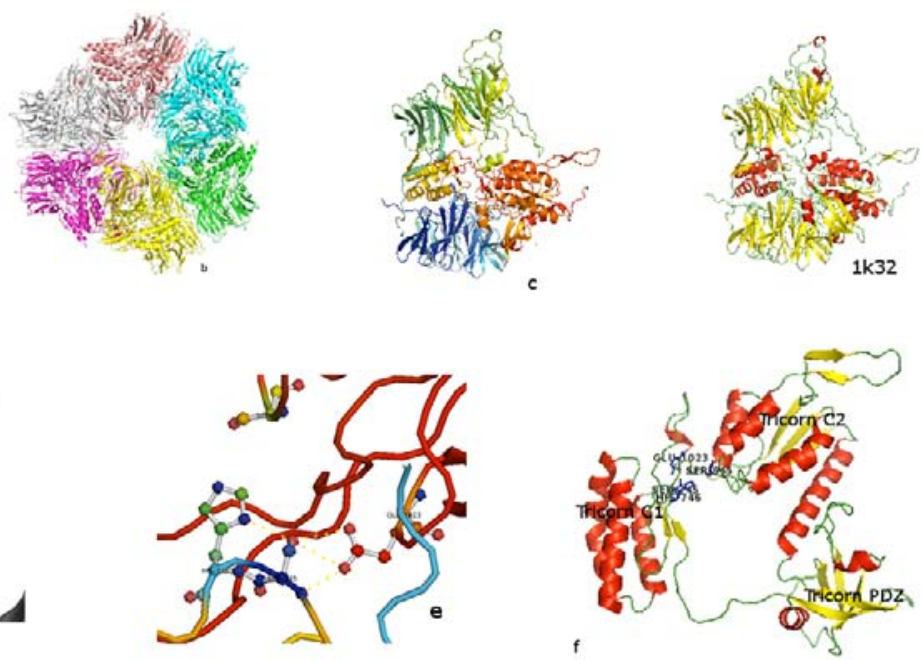

Figure 1: a; The hexameric 3D structure of Thermoplasma acidophilum tricorn protease coloured by chain, $b$; the oriented form of ' $a$ ' showing all the six chains, $c$ : tricorn protease monomer coloured by spectrum from $\mathrm{N}$ - to $\mathrm{C}$-terminal domain where blue reprsents the $\mathrm{N}$-terminal domain (6-bladed beta propeller), green - tricorn protease domain 2 (7-bladed beta propeller), orange- PDZ domain, red: C-terminal domain, d: surface representation of tricorn protease active site with active site residues shown as purple sticks while the the main chain shown as ribbon, e: active site tetrad shown as ball and sticks, f; tricorn protease C-terminal domain, arranged as C1, PDZ, C2 in that order in the polypeptide chain.
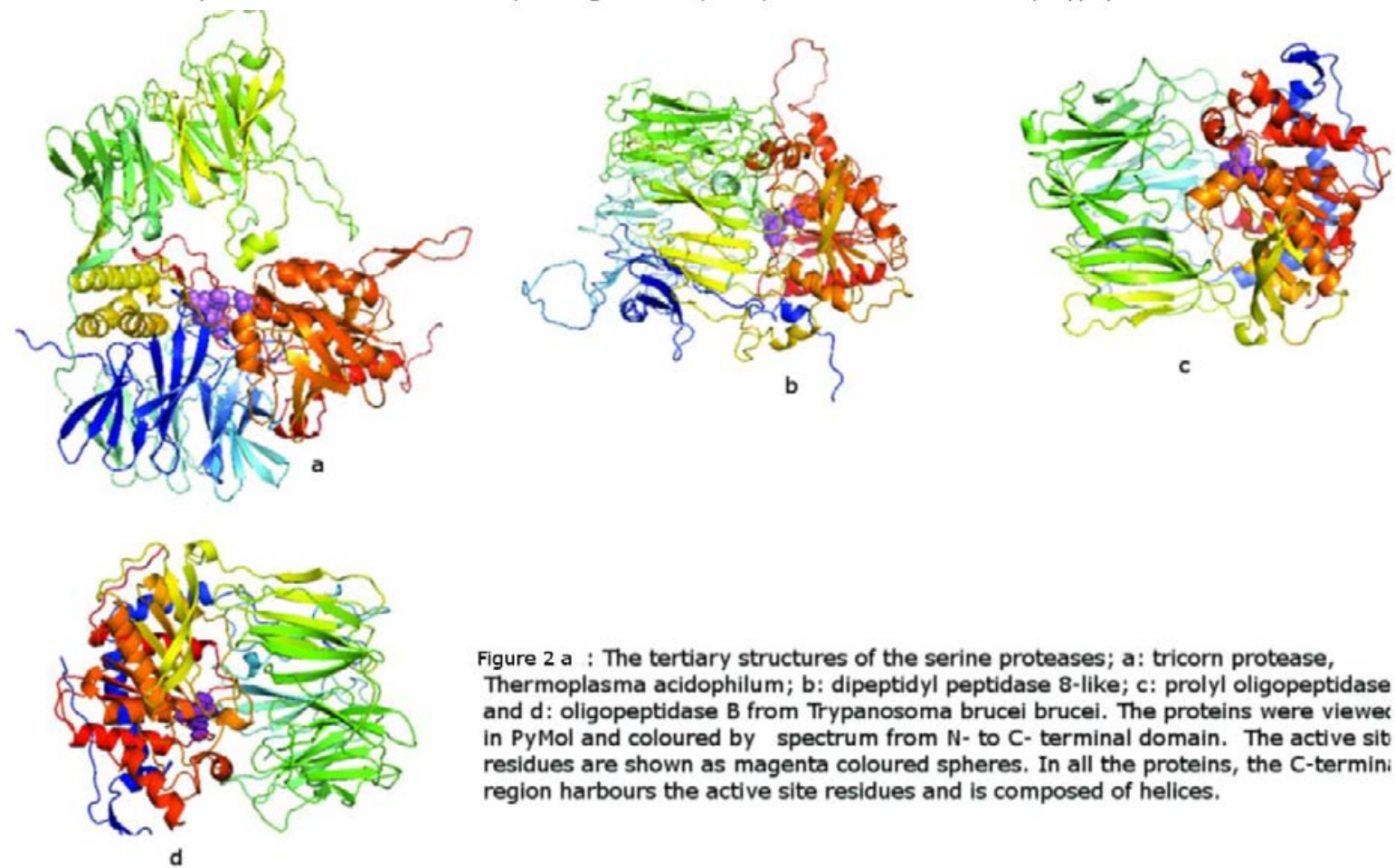

Figure 2 a : The tertiary structures of the serine proteases; a: tricorn protease, Thermoplasma acidophilum; b: dipeptidyl peptidase 8-like; c: prolyl oligopeptidase and d: oligopeptidase B from Trypanosoma brucei brucei. The proteins were viewe in PyMol and coloured by spectrum from $\mathrm{N}$ - to $\mathrm{C}$ - terminal domain. The active sit residues are shown as magenta coloured spheres. In all the proteins, the C-termini region harbours the active site residues and is composed of helices.

Volume 5 Issue 7, July 2016 www.ijsr.net 


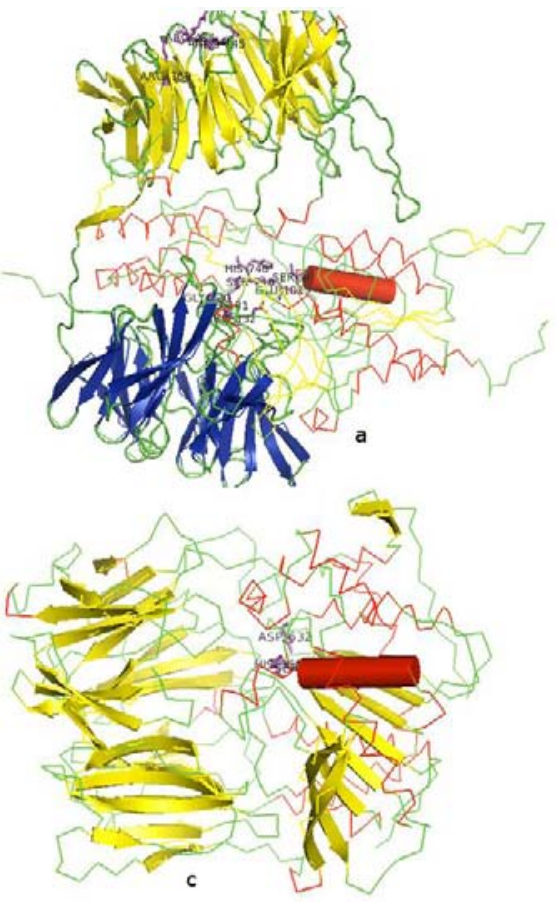

Figure 2b: The structural homology of the catalytic core element, serine following the strictly conserved helix shown as red cylindrical helix in the structure. The main chain is shown as ribbon coloured by secondary structure where red, yellow and green represents helices, sheets and coils respectively. The beta propeller sheets provide substrate entrance and exit routes to/from the active site. In tricorn protease ( $T$. acidophilum), the serine is at position 965 as shown on "a"; S699, S549 and S563 in dipeptidyl peptidase IV, prolyl oligopeptidase and oligopetidase B (b, c and d) respectively. This is a striking similarity in these proteins which suggests a functional relationship.
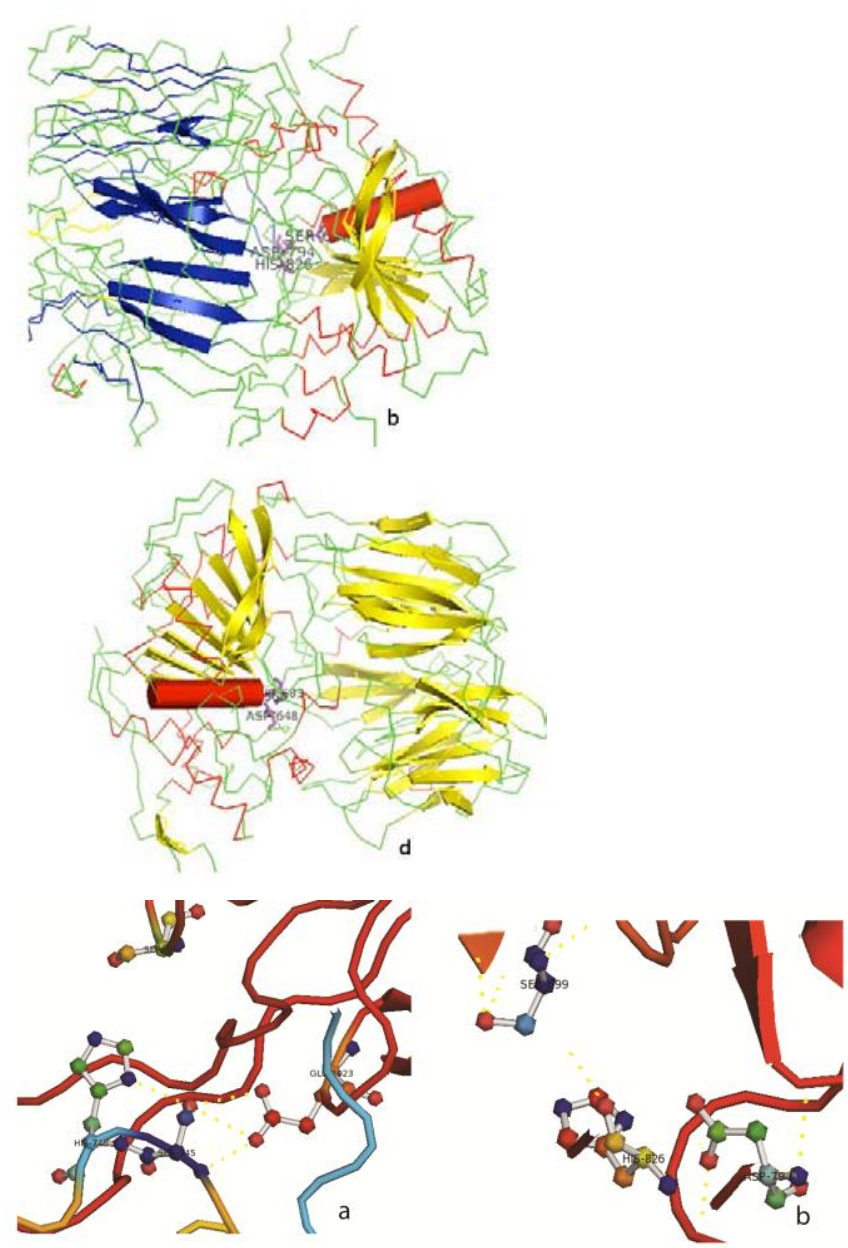

Figure $2 \mathrm{c}$ : The orientation of the active site residues; a: tricorn protease and b: dipeptidyl peptidase 8 -like which was chosen to represent the other trypanosome proteins due to their similarity in orientation.

Comparative genomics of serine peptidase in the two organisms in this study points out to unequal distribution of the serine peptidases where S41 seemed to lack in trypanosomes which seemed to have S8 and S9 peptidases (Appendix 1).

Appendix 1: Distribution of serine peptidase families in Thermoplasma acidophilum and Trypanosoma brucei brucei

\begin{tabular}{|c|c|c|c|}
\hline MEROPS ID & Name & T. acidophilum & T. brucei brucei \\
\hline S8 unassigned & subfamily S8A unassigned peptidases & 0 & 2 \\
\hline S09.A29 & At4g17150 (Arabidopsis thaliana)-type peptidase & 0 & 1 \\
\hline S9 homologues & family S9 non-peptidase homologues & 0 & 1 \\
\hline S9 unassigned & subfamily S9A unassigned peptidases & 0 & 4 \\
\hline S9 unassigned & subfamily S9C unassigned peptidases & 0 & 3 \\
\hline S9 unassigned & family S9 unassigned peptidases & 1 & 2 \\
\hline S10 unassigned & family S10 unassigned peptidases & 0 & 3 \\
\hline S16.A11 & PF0467 (Pyrococcus furiosus) & 1 & 0 \\
\hline S26 unassigned & subfamily S26A unassigned peptidases & 0 & 1 \\
\hline S26 unassigned & subfamily S26B unassigned peptidases & 0 & 1 \\
\hline S33.005 & tricorn interacting factor F1 & 1 & 0 \\
\hline S33.010 & SCO7095-type peptidase & 1 & 5 \\
\hline S33 unassigned & family S33 unassigned peptidases & 1 & 0 \\
\hline S41.005 & tricorn core peptidase (archaea) & 2 & 0 \\
\hline S45 unassigned & family S45 unassigned peptidases & 1 & 0 \\
\hline S49 homologues & subfamily S49B non-peptidase homologues & 1 & 0 \\
\hline S49 unassigned & subfamily S49A unassigned peptidases & 2 & 0 \\
\hline S53 unassigned & family S53 unassigned peptidases & 0 & 1 \\
\hline S59 homologues & family S59 non-peptidase homologues & 1 & 0 \\
\hline S66.001 & murein tetrapeptidase LD-carboxypeptidase & & 0 \\
\hline
\end{tabular}

Volume 5 Issue 7, July 2016 www.ijsr.net 


\section{International Journal of Science and Research (IJSR) \\ ISSN (Online): 2319-7064 \\ Index Copernicus Value (2013): 6.14 | Impact Factor (2015): 6.391}

The S8 and S9 are well developed in T. brucei brucei while the $\mathrm{S} 41$ is well developed in T. acidophilum.

Studies have also shown that most of the higher eukaryotic organisms from yeast to mammals, utilize proteasomes as well as large enzyme complexes such as the cytosolic and lysosomal dipeptidyl and tripeptidyl peptidases (DPP and TPP) with functional analogies to tricorn (Tomkinson 1999, Geier, et al., 1999). Studies have also shown that tricorn protease is patchily distributed and other archaeon such as Desulforococcales, example Pyrolobus, Desulforococcus lack tricorn protease but have tetrahedral aminopeptidase (TET) which also acts downstream of the proteasome assumes the role of tricorn protease (Borissenko \& Groll 2005).

\section{Conclusion}

Based on the structural similarities, this study proposes the T. b. brucei dipeptidyl peptidase IV, oligopeptidase B and prolyl oligopeptidase as tricorn protease functional analogs.

\section{Declaration}

The authors declare that there is no conflict of interests regarding the publication of this paper.

\section{Acknowledgements}

The authors would like to the Jomo Kenyatta University of Agriculture and Technology, Research Production and Extension division and National Commission for Science, Technology and Innovation (NACOSTI/RCD/ST \& I $5^{\text {th }}$ CALL PhD/145) for funding this project.

\section{References}

[1] Bastos, I.M.D. et al., 2010. Prolyl oligopeptidase of Trypanosoma brucei hydrolyzes native collagen, peptide hormones and is active in the plasma of infected mice. Microbes and Infection, 12(6), pp.457-466. Available at: http://www.sciencedirect.com/science/article/pii/S1286 457910000559.

[2] Borissenko, L. \& Groll, M., 2005. Crystal structure of TET protease reveals complementary protein degradation pathways in prokaryotes. Journal of molecular biology, 346(5), pp.1207-19. Available at: http://www.sciencedirect.com/science/article/pii/S0022 283605000045

[3] Brandstetter, H. et al., 2001. Crystal structure of the tricorn protease reveals a protein disassembly line. Nature, 414(6862), pp.466-470.

[4] Delano, W.L. \& Ph, D., 2004. PyMOL: Molecular graphics system. Available at https://www.pymol.org/

[5] Franco, J.R. et al., 2014. Epidemiology of human African trypanosomiasis. Clinical epidemiology, 6, pp.257-75.

[6] Groll, M. et al., 2005. Molecular machines for protein degradation. ChemBioChem, 6(2), pp.222-256.

[7] Huang, T.-T. et al., 2015. (PS)2: protein structure prediction server version 3.0. Nucleic Acids Research,
43(May), pp.338-342. Available at: http://nar.oxfordjournals.org/lookup/doi/10.1093/nar/gk v454.

[8] Katoh, K. \& Standley, D.M., 2013. MAFFT multiple sequence alignment software version 7: Improvements in performance and usability. Molecular Biology and Evolution, 30(4), pp.772-780.

[9] Kim, J.S. et al., 2002. Navigation inside a protease: substrate selection and product exit in the tricorn protease from Thermoplasma acidophilum. Journal of Molecular Biology, 324(5), pp.1041-1050. Available at: papers://ae875177-834e-4ba8-8523120292c79891/Paper/p3736.

[10] Klemba, M. \& Goldberg, D.E., 2002. Biological roles of proteases in parasitic protozoa. Annual Review of Biochemistry, 71(1), pp.275-305. Available at: http://www.annualreviews.org/doi/abs/10.1146/annurev. biochem.71.090501.145453.

[11] Marchler-Bauer, A. et al., 2015. CDD: NCBI's conserved domain database. Nucleic Acids Research, 43(D1), pp.D222-D226.

[12] Pallen, M.J., Lam, A.C. \& Loman, N., 2001. Tricornlike proteases in bacteria. Trends in Microbiology, 9(11), pp.518-521. Available at: http://dx.doi.org/10.1016/S0966-842X(01)02199-0.

[13] Simarro, P.P. et al., 2011. The human african trypanosomiasis control and surveillance programme of the world health organization 2000-2009: The way forward. PLoS Neglected Tropical Diseases, 5(2).

[14] Simo, G. et al., 2014. Challenges towards the elimination of Human African Trypanosomiasis in the sleeping sickness focus of Campo in southern Cameroon. Parasites \& Vectors, 7(1), p.374. Available at: http://www.parasitesandvectors.com/content/7/1/374.

[15] Tamura, T. et al., 1996. Tricorn protease--the core of a modular proteolytic system. Science (New York, N.Y.), 274(5291), pp.1385-1389.

[16] Tomkinson, B., 1999. Tripeptidyl peptidases: enzymes that count. Trends in Biochemical Sciences, 24(9), pp.355-359. Available at: http://dx.doi.org/10.1016/S0968-0004(99)01435-8.

[17] Waterhouse, A.M. et al., 2009. Jalview Version 2-A multiple sequence alignment editor and analysis workbench. Bioinformatics, 25(9), pp.1189-1191. 